\title{
Evaluating the Effectiveness of Diabetes Shared Medical Appointments (SMAs) as Implemented in Five Veterans Affairs Health Systems: a Multi-site Cluster Randomized Pragmatic Trial
}

\author{
Michele Heisler, MD, MPA ${ }^{1,2,3,4}$, Jennifer Burgess, $M P H^{7}$, Jeffrey Cass, PsyD ${ }^{5}$, \\ John F. Chardos, MD 6 , Alexander B. Guirguis, PharmD, BCPS , Lorrie A. Strohecker, MD ${ }^{5}$, \\ Adam S. Tremblay, $M D^{1,2}$, Wen-Chih Wu, $\mathrm{MD}^{8}$, and Donna M. Zulman, $M D, M S^{6,9}$
}

\begin{abstract}
${ }^{7}$ Veterans Affairs Center for Clinical Management Research, VA Ann Arbor Healthcare System, Ann Arbor, MI, USA; ${ }^{2}$ Department of Internal Medicine, University of Michigan Medical School, Ann Arbor, MI, USA; ${ }^{3}$ Department of Health Behavior and Health Education, School of Public Health, University of Michigan, Ann Arbor, MI, USA; ${ }^{4}$ North Campus Research Complex, University of Michigan, Ann Arbor, MI, USA; ${ }^{V}$ A Northern California Health Care System, Mather, CA, USA; ${ }^{\circ}$ Veterans Affairs Center for Innovation to Implementation, VA Palo Alto Health Care System, Palo Alto, CA, USA; ${ }^{7}$ VA Connecticut Healthcare System, West Haven, CT, USA; ${ }^{8}$ Providence VA Medical Center, Providence, RI, USA; ${ }^{9}$ Division of General Medicine Disciplines, Stanford University, Stanford, CA, USA.
\end{abstract}

OBJECTIVE: To examine whether diabetes shared medical appointments (SMAs) implemented as part of usual clinical practice in diverse health systems are more effective than usual care in improving and sustaining Alc improvements.

RESEARCH DESIGN AND METHODS: A multi-site cluster randomized pragmatic trial examining implementation in clinical practice of diabetes SMAs in five Veterans Affairs (VA) health systems was conducted from 2016 to 2020 among 1537 adults with type 2 diabetes and elevated Alcs. Eligible patients were randomly assigned to either: (1) invitation to participate in a series of SMAs totaling 8-9 h; or (2) continuation of usual care. Relative change in Alc (primary outcome) and in systolic blood pressure, insulin starts, statin starts, and anti-hypertensive medication classes (secondary outcomes) were measured as part of usual clinical care at baseline, at 6 months and at 12 months ( $\sim 7$ months after conclusion of the final SMA in four of five sites). We examined outcomes in three samples of SMA participants: all those scheduled for a SMA, those attending at least one SMA, and those attending at least half of SMAs.

RESULTS: Baseline mean Alc was 9.0\%. Participants scheduled for an SMA achieved Alc reductions $0.35 \%$ points greater than the control group between baseline and 6-months follow up $(p=.001)$. Those who attended at least one SMA achieved reductions $0.42 \%$ points greater $(p<.001)$, and those who attended at least half of scheduled SMAs achieved reductions $0.53 \%$ points greater $(p<.001)$ than the control group. At 12-month follow-up, the three SMA analysis samples achieved reductions from baseline ranging from $0.16 \%$ points $(p=0.12)$ to $0.29 \%$ points ( $p=.06$ ) greater than the control group.

Received June 18, 2020

Accepted December 29, 2020

Published online February 2, 2021
CONCLUSIONS: Diabetes SMAs as implemented in reallife diverse clinical practices improve glycemic control more than usual care immediately after the SMAs, but relative gains are not maintained. Our findings suggest the need for further study of whether a longer term SMA model or other follow-up strategies would sustain relative clinical improvements associated with this intervention.

TRIAL REGISTRATION: ClinicalTrials.gov ID NCTO2 132676

KEY WORDS: shared medical appointment, peer support, disease management, implementation, diabetes mellitus, pragmatic clinical trial.

$J$ Gen Intern Med 36(6):1648-55

DOI: $10.1007 / \mathrm{s} 11606-020-06570-\mathrm{y}$

(c) This is a U.S. government work and not under copyright protection in the U.S.; foreign copyright protection may apply 2021

\section{INTRODUCTION}

Health burdens and costs of type 2 diabetes mellitus ( T 2 DM) - a leading cause of morbidity and mortality - continue to soar. One of three US adults without diabetes at age 45 is projected to develop T2DM. ${ }^{1}$ Success of diabetes treatments depends on patients' initiating and sustaining key behaviors - taking medications, eating healthily, being physically active, self-monitoring. Many patients need self-management support. ${ }^{2,3}$ Health systems thus seek models to improve diabetes self-management support and clinical management that are more low-cost and scalable than offering frequent one-on-one visits with providers.

One potentially effective and efficient model for providing integrated medical care and self-management support is diabetes shared medical appointments (SMAs). SMAs bring groups of patients together with an interdisciplinary team of providers for a series of 60-120-min sessions. Session leaders encourage participants to set behavioral goals and steps to meet these goals ("action planning"), ${ }^{4}$ discuss key areas of 
diabetes care, and encourage participants to share experiences and self-management strategies with each other. ${ }^{5,6}$ One team provider has prescribing privileges and meets individually with participants to adjust medications.

Some efficacy trials have found SMAs to be more effective than usual care in improving A1c. ${ }^{7-11}$ In Edelman et al.'s 2010 trial, group medical visits for adults with both high A1cs and blood pressure improved blood pressure but not A1cs. ${ }^{12}$ Meta-analyses have reinforced the efficacy of SMAs, ${ }^{13-16}$ though one found that their heterogeneity does not allow conclusions about which SMA components are necessary for effectiveness. ${ }^{13}$ They emphasized the need now for SMAs to be evaluated as implemented in real-life clinical practice. It is also necessary to examine longer term outcomes after the conclusion of SMAs offered to participants over a limited period, as is the case in most programs. One study examining outcomes 13-18 months after the conclusion of the 2010 Edelman et al. trial found that blood pressure gains relative to the control group were not maintained, but participants had lower rates of hospital inpatient admissions and expenditures.

${ }^{17}$ However, most studies to date have examined outcomes immediately after participation in SMAs. More research is needed to understand if benefits are sustained after participants return to usual care.

Novel approaches such as mobilizing peer support among SMA participants may help patients sustain improvements achieved through SMAs. Efficacy trials have found that reciprocal peer support programs involving telephone calls between paired participants and periodic peer-led group sessions improve glycemic control more than nurse care management. ${ }^{18}$ It is not known whether patients in real-life SMAs would be interested in participating in such a program and, if so, whether participation would sustain improvements better than a return to usual care alone. Again, whether what is effective in controlled clinical trials led by well-trained research staff is equally effective when implemented in usual clinical practice is unknown.

To address these gaps in knowledge, we worked with five geographically diverse VA health systems implementing diabetes SMAs as part of clinical practice to establish a core set of SMA elements and to develop a rigorous evaluation of these SMAs' effectiveness. We also sought to examine whether SMA participants offered the opportunity at their first SMA session to pair up with another participant to support each other would sustain improved clinical outcomes more than participants in SMA cohorts not offered this option. We thus conducted a multi-site cluster randomized trial to examine effectiveness of SMAs as implemented in diverse real-life VA settings in improving glycemic control (primary outcomes) and systolic blood pressure (SBP), insulin starts, statin starts, and anti-hypertensive medication class changes (secondary outcomes) compared with usual care. We also examined the feasibility and effectiveness of offering a reciprocal peer support program (P2P) as a complement to participation in the SMAs.

\section{RESEARCH DESIGN AND METHODS}

\section{Setting}

Since 2005, the VA has mandated SMAs, yet had not evaluated their effectiveness as implemented compared with usual care. For this evaluation, we enlisted the support of clinical leadership at five VA health care systems implementing diabetes SMAs (Table 1).

\section{Patient Selection, Recruitment, and Randomization}

The study protocol is described elsewhere. ${ }^{19}$ Briefly, from May 2016 to May 2018, we identified from electronic health records (EHR) patients who had (1) two outpatient visits or one hospitalization with a diabetes-related ICD-10 code in the prior 12 months; or (2) at least one prescription for a glucose control medication; and (3) an A1c $\geq 7.5 \%$ if age $<70$ or $\geq$ $8.0 \%$ if age $70+$ years within 6 months prior to enrollment. We excluded patients with active substance abuse disorders, schizophrenia, severe dementia, or bipolar disorder.

As both SMAs and P2P were clinical program offerings, analysis of clinical outcomes among participants did not require Central VA Institutional Review Board (CIRB) oversight. The CIRB granted a waiver of signed consent to allow us to evaluate clinical outcomes from the EHR for all participants.

Patients were identified to be randomized through quarterly data pulls from VA clinical databases. As there were more eligible participants at each site than capacity, patients identified through the EHR each quarter (and also through provider referrals at one site) were randomized by random number generator to usual care or to be invited to participate in SMAs. Some SMA cohorts were then randomly selected for participants to be offered at their first SMA session the opportunity to participate in P2P.

\section{Usual Care}

The study team had no contact with the group who received standard VA services. Eligible patients randomized to usual care were distributed equally across the recruitment period.

\section{SMA-Only Program}

As the aim was to evaluate real-world effectiveness, the sites were given latitude in their SMAs' frequency, duration, and content. All sites, however, agreed to key shared SMA elements (see Table 1). These elements were selected based on study team expertise, the literature on components found in effective SMAs, and hypothesized mechanisms for effectiveness. $^{20-25}$

\section{SMA + Reciprocal Peer Support Program}

The P2P program was offered at the first SMA session to some SMA groups randomized to SMA+P2P. Participants who agreed to participate were matched with another SMA 
Table 1 Health Care Facility and Shared Medical Appointment (SMA) Information

\begin{tabular}{|c|c|c|c|c|c|}
\hline & Ann Arbor & Palo Alto* & Providence & Sacramento & $\begin{array}{l}\text { West } \\
\text { Haven }\end{array}$ \\
\hline $\begin{array}{l}\text { Fiscal year 2016: number of type } 2 \text { diabetes seen in } \\
\text { ambulatory care with eligible A1c }\end{array}$ & 11,671 & 14,545 & 9017 & 9407 & 9367 \\
\hline \multirow{11}{*}{$\begin{array}{l}\text { Shared medical appointment (SMA) clinician team trained } \\
\text { in motivational interviewing-based group facilitation and } \\
\text { action planning }\end{array}$} & Doctor of & PharmD & PharmD & $\mathrm{MD}$ & PharmD \\
\hline & Pharmacy & Health Behavior & $\mathrm{HBC}$ & PharmD & $\mathrm{HBC}$ \\
\hline & (PharmD) & Coordinator & $\mathrm{MD}$ & $\mathrm{HBC}$ & RD \\
\hline & Registered & $(\mathrm{HBC})$ & $\mathrm{RD}$ & $\mathrm{RD}$ & RN \\
\hline & Dietician (RD) & $\mathrm{RD}$ & $\mathrm{RN}$ & & $\mathrm{MD} \dagger$ \\
\hline & Registered Nurse & $\mathrm{RN}$ & & & \\
\hline & $(\mathrm{RN})$ & $\mathrm{MD}^{\dagger}$ & & & \\
\hline & $\begin{array}{l}\text { Medical Doctor } \\
(\mathrm{MD})^{\dagger}\end{array}$ & $\begin{array}{l}\text { Social Worker } \\
(\mathrm{SW})^{\dagger}\end{array}$ & & & \\
\hline & Licensed Practical & Registered Nurse & & & \\
\hline & Nurse $(\mathrm{LPN})^{\dagger}$ & Practitioner $(\mathrm{RNP})^{\dagger}$ & & & \\
\hline & & $\begin{array}{l}\text { Physical Therapist } \\
(\mathrm{PT})^{\dagger}\end{array}$ & & & \\
\hline Frequency of SMAs/cohort & Monthly & Biweekly & Biweekly & Weekly & Quarterly \\
\hline \# of SMAs/cohort & 4 & 4 & 6 & 8 & \\
\hline Duration of each SMA & $2 \mathrm{~h}$ & $2 \mathrm{~h}$ & $1.5 \mathrm{~h}$ & $1 \mathrm{~h}$ & $2 \mathrm{~h}$ \\
\hline Total SMA dose/cohort & $8 \mathrm{~h}$ & $8 \mathrm{~h}$ & $9 \mathrm{~h}$ & $8 \mathrm{~h}$ & $8 \mathrm{~h}$ \\
\hline Mean SMA cohort size & 7.15 & 8.69 & 8.85 & 6.15 & 5.08 \\
\hline Range of SMA cohort size & $5-10$ & $5-13$ & $5-11$ & $3-19$ & $2-10$ \\
\hline $\begin{array}{l}\text { All sessions include (1) review of participants' vitals and } \\
\text { labs; (2) action planning (goal setting) and discussion of } \\
\text { each participant's progress, challenges, strategies to meet } \\
\text { action step and formulation of new step; and (3) prescriber } \\
\text { holds brief individual sessions with each participant to } \\
\text { review medications and make medication changes as } \\
\text { necessary. }\end{array}$ & Yes & Yes & Yes & Yes & Yes \\
\hline $\begin{array}{l}\text { Group facilitation focused on creating patient-driven, } \\
\text { interactive discussion among participants }\end{array}$ & Yes & Yes & Yes & Yes & Yes \\
\hline $\begin{array}{l}\text { Information and education on medications, blood pressure } \\
\text { and lipid control, diet, exercise, stress management that is } \\
\text { driven by participants' interests and questions } \ddagger\end{array}$ & Yes & Yes & Yes & Yes & Yes \\
\hline
\end{tabular}

*Includes Palo Alto, Livermore, Fremont, and San Jose

†Occasional participant/guest speaker

$¥$ To assess fidelity of SMA sessions across all sites, a trained research staff member attended all SMA sessions for a subset of cohorts at each site and completed a fidelity checklist

participant and encouraged to make weekly phone calls with each other between SMA sessions and after completion of the SMA series. They also were offered peer facilitator-led group sessions focused on participants' diabetes self-management goals every 4-6 weeks for a minimum of 12 months following enrollment in SMAs.

\section{Data Collection and Outcomes Measures}

Our primary outcome was the difference between mean baseline A1c values in 6-month windows preceding baseline and following the 6- and 12-month post-enrollment evaluation periods. Secondary outcomes included change in systolic blood pressure (SBP), anti-hypertensive medication use, statin use, and insulin starts across the same evaluation periods. We hypothesized that SMA participants would be more likely to start insulin and a statin and to have changes in antihypertensive medications than those in usual care.

We obtained A1c and SBP values entered in the EHR closest to each timepoint. Data on medication use were obtained through the VHA Corporate Data Warehouse. The 6month evaluation period was $\geq 3$ to $<9$ months postenrollment and the 12-month evaluation period was $\geq 9$ to $<15$ months post-enrollment.

\section{Sample Size Power Calculations}

We estimated we could conduct 10 to 16 SMA groups per site with 8-14 people per group. At a minimum, this would equate to 560 people in active treatment and an equal number of controls. In this scenario, we could detect a $0.5 \%$ difference in A1c and a 5-mmHg decline in SBP between groups.

\section{Analyses}

Our primary analysis was an intention to treat analysis (ITT) comparing all eligible participants randomized to be scheduled for a SMA (regardless of whether they attended a SMA) or to usual care. We also conducted analyses comparing outcomes between the usual care group and (1) the attendee group, defined as patients who attended at least one SMA, and (2) the engagement group, defined as patients who attended at least half of offered SMAs.

We conducted a "difference-in-differences" (DID) analysis of A1c and SBP changes using a multilevel linear mixed effect model. ${ }^{26,27}$ The model included a treatment indicator, a time indicator, the treatment-time interaction (DID estimator), and baseline A1c, age, gender, and race. Random intercepts were included for patients (level-1) nested within site (level-2). In 
analyses of insulin and statin starts, we implemented a multilevel logistic regression model, and for anti-hypertensive medication class changes, we implemented a multilevel Poisson regression model. ${ }^{28,29}$

As a robustness check, we repeated analyses on a subset of patients matched 1:1 based on propensity scores. ${ }^{30}$ The propensity score model was developed using a logistic regression on patient demographics. Although our initial analyses used observed data, because we were relying on data available in the EHR, we anticipated more missing values than if this were an efficacy trial. Accordingly, we used logistic regression to model patients' likelihood of having outcome data and defined strata within which outcome values were missing at random. We then stratified patients according to these propensities and randomly sampled from the observed outcome distribution and imputed these values for missing data within each stratum. In sensitivity analyses, we (1) imputed missing data such that any missing A1c measurements at 6 or 12 months would match the value at baseline, ${ }^{31}$ (2) examined outcomes among a subset of patients with full outcomes data, and (3) used multiple imputation to impute missing A1cs, race, and age. ${ }^{32}$ We used the BenjaminiHochberg procedure to control for multiple comparisons. ${ }^{33}$

Because only 59 patients in the SMA+P2P group chose to participate in $\mathrm{P} 2 \mathrm{P}$, we did not have statistical power to compare outcomes between the SMA-only and SMA+P2P arms. Therefore, we conducted our primary analysis that combined the two SMA groups (SMA-only and SMA+P2P) into one active treatment group and compared clinical outcomes of these patients with those in usual care. In an exploratory analysis, we examined changes in A1c among the $59 \mathrm{P} 2 \mathrm{P}$ participants.

\section{RESULTS}

\section{Participant Flow and Baseline Data}

The CONSORT diagram (Fig. 1) shows participant flow. Participants were more likely than non-participants to be nonwhite, have higher levels of education, report poorer health,

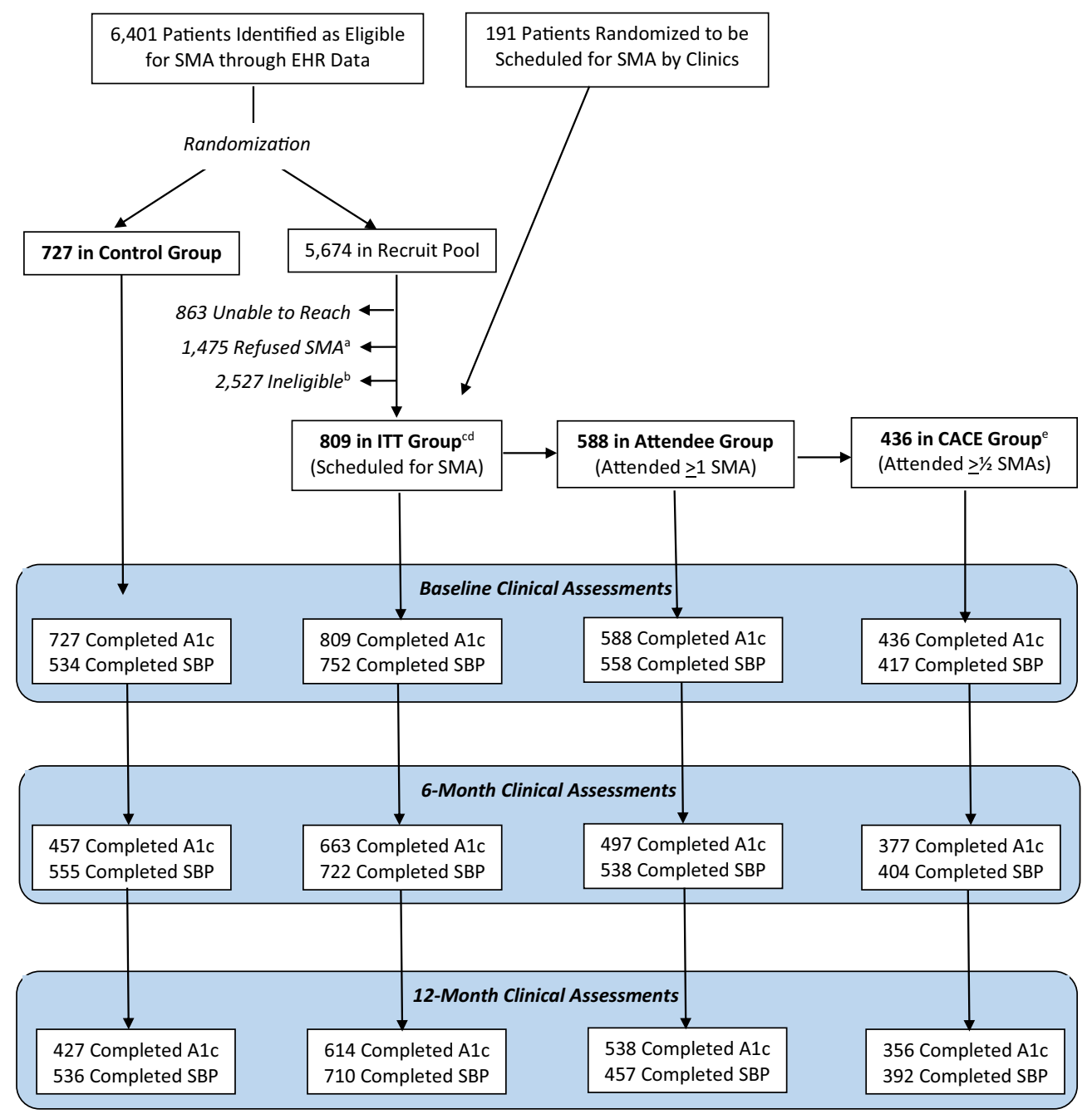

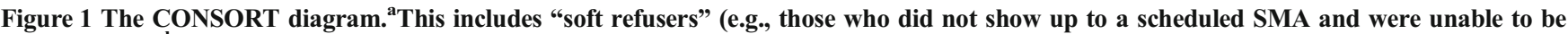
rescheduled). "This includes those whose A1c's "expired" ( $\geq 6$ months old) before they could be recruited for an SMA. "Of these, 304 were offered the P2P program and, of those, 59 actively participated in the P2P program. ${ }^{\mathrm{d}} \mathrm{Of}$ these, 451 completed a baseline survey, 376 completed a 6-month survey, and 348 completed a 12-month survey. ${ }^{~} \mathrm{CACE}$ (Complier Average Causal Effect) analysis consists of those meeting our prespecified threshold for effective engagement 
be less likely to be on insulin, and report less satisfaction with their level of social support. 810 (35\% of contacted patients) were scheduled to attend a SMA. Of these, 588 attended at least 1 SMA (73\%), and $436(54 \%)$ attended half or more of SMAs, a level of engagement defined a priori as likely to be an adequate dose of participation. (See Appendix 1 for chi-square analyses comparing characteristics between groups and between participants with differing levels of SMA engagement.)

Participants' baseline characteristics are reported in Table 2. We had complete 6-month outcome data on 457 control (63\%) and 663 SMA participants (82\%) and complete 12-month data on 427 control $(60 \%)$ and 614 SMA participants (76\%).

\section{Changes in Primary Outcome of Alc}

SMA participants achieved clinically and statistically significant greater reductions in A1c than those in the control group between baseline and 6 months, but differences between groups were no longer statistically significant at 12 months (Table 3). Participants scheduled for a SMA achieved A1c reductions $0.35 \%$ points greater than the control group between baseline and 6-months follow up $(p=.001)$. Those who attended at least one SMA session achieved reductions of $0.42 \%$ points greater $(p<.001)$, and those who attended at

Table 2 Baseline Characteristics of Intervention and Control Group Patients

\begin{tabular}{|c|c|c|}
\hline \multirow[t]{2}{*}{ Characteristic } & $\begin{array}{l}\text { Intervention }(N \\
=809)\end{array}$ & $\begin{array}{l}\text { Control }(N \\
=727)\end{array}$ \\
\hline & \multicolumn{2}{|c|}{$N(\%)$ or mean $\pm \mathrm{SD}$} \\
\hline Age (years) as of $6 / 1 / 18$ & $67.1 \pm 9.2$ & $67.8 \pm 12.7$ \\
\hline $\begin{array}{l}\text { Male } \\
\text { Race* }\end{array}$ & $782(97 \%)$ & 707 (97\%) \\
\hline \multicolumn{3}{|l|}{ Race* } \\
\hline White & $550(68 \%)$ & $540(74 \%)$ \\
\hline Black & $150(19 \%)$ & $89(12 \%)$ \\
\hline Asian & $29(4 \%)$ & $26(4 \%)$ \\
\hline American Indian/Alaskan Native & $8(1 \%)$ & $7(1 \%)$ \\
\hline Hawaiian/Pacific Islander & $20(2 \%)$ & $11(2 \%)$ \\
\hline Missing & $60(7 \%)$ & $61(8 \%)$ \\
\hline \multicolumn{3}{|l|}{ Ethnicity } \\
\hline Hispanic or Latino & $70(9 \%)$ & $64(9 \%)$ \\
\hline Not Hispanic or Latino & $702(87 \%)$ & $626(86 \%)$ \\
\hline Missing & $37(5 \%)$ & $37(5 \%)$ \\
\hline $\begin{array}{l}\text { Most recent hemoglobin A1c in } \\
\text { the last } 8 \text { months }(\%)\end{array}$ & $9.1 \pm 1.5$ & $8.9 \pm 1.3$ \\
\hline $\begin{array}{l}\text { Mean systolic blood pressure over } \\
\text { the last } 8 \text { months }(\mathrm{mmHg})\end{array}$ & $136.2 \pm 13.4$ & $137.7 \pm 15.2$ \\
\hline $\begin{array}{l}\text { Most recent systolic blood } \\
\text { pressure in the last } 8 \text { months } \\
(\mathrm{mmHg})\end{array}$ & $137.2 \pm 17.5$ & $139.6 \pm 18.9$ \\
\hline On insulin & $385(48 \%)$ & $255(35 \%)$ \\
\hline On statin & $603(75 \%)$ & $470(65 \%)$ \\
\hline $\begin{array}{l}\text { Classes of antihypertensive } \\
\text { medications }\end{array}$ & $2.4 \pm 1.2$ & $2.2 \pm 1.3$ \\
\hline $\begin{array}{l}\text { Primary care in-person visits in } \\
\text { past } 8 \text { months }\end{array}$ & $3.4 \pm 3.1$ & $3.2 \pm 3.4$ \\
\hline $\begin{array}{l}\text { Primary care phone visits in past } \\
8 \text { months }\end{array}$ & $0.7 \pm 1.2$ & $0.7 \pm 1.5$ \\
\hline $\begin{array}{l}\text { Nurse Case Manager in-person } \\
\text { visits in past } 8 \text { months }\end{array}$ & $0.2 \pm 0.7$ & $0.2 \pm 0.8$ \\
\hline $\begin{array}{l}\text { Nurse Case Manager phone visits } \\
\text { in past } 8 \text { months }\end{array}$ & $0.3 \pm 0.9$ & $0.3 \pm 0.9$ \\
\hline $\begin{array}{l}\text { Endocrinology in-person visits in } \\
\text { past } 8 \text { months }\end{array}$ & $0.3 \pm 0.8$ & $0.2 \pm 0.8$ \\
\hline
\end{tabular}

*Patients can have more than 1 race listed, so these do not add to $100 \%$ least half of scheduled sessions achieved reductions of $0.53 \%$ points greater $(p<.001)$ than the control group. At 12-month follow-up, the three SMA samples achieved reductions from baseline ranging from $0.16 \%$ points $(p=0.12)$ to $0.29 \%$ points $(p=.06)$ greater than the control group, none of which were significant at the $p<.05$ level. The $59 \mathrm{P} 2 \mathrm{P}$ participants improved in A1c from a mean of $8.89 \%$ to $8.15 \%$ at 6 months and $8.25 \%$ at 12 months. All sensitivity analyses addressing missing data were similar to our main analysis and showed the same dose-response effects (see Appendix 2). Similarly, A1c improvements in the SMA group were unchanged when P2P participants were excluded from analyses.

\section{Secondary Outcomes}

There were no significant differences in SBP changes between the control group and any of the three SMA analytical samples at 6 months or 12 months (Table 3 ). There were significantly more insulin and statin starts in all three SMA samples at 6 months and 12 months than in the control group (Table 3). There were no differences in changes in anti-hypertensive medication classes between groups.

\section{DISCUSSION}

This study contributes to the literature by providing data on the effectiveness of SMAs as implemented as part of normal clinical practice. Across five geographically diverse VA health systems, diabetes patients with high A1cs who were scheduled for a SMA, who attended at least one SMA, and who attended at least half of SMAs all achieved statistically and clinically significant reductions in A1c at six-months follow-up that were greater than patients in usual care. There was a dose response in effects: Patients who attended at least half of scheduled SMAs achieved A1c reductions $0.11 \%$ points greater than patients who attended at least one SMA and $0.18 \%$ points greater than those scheduled for a SMA. At 12 months, both intervention and control groups achieved clinically and statistically significant A1c reductions from baseline, with no significant differences between groups. Mean A1c reductions from baseline to 12 months ranged from 0.95 to $1.08 \%$ points for patients in the SMA groups. A mean difference in A1c level of $0.5 \%$ translates into an absolute $2.8 \%$ risk reduction in diabetes events over 10 years, or a number needed to treat of 36 (i.e., of 36 treated, one person with reduction in diabetes events). ${ }^{34}$

SMA participants maintained more insulin starts and statin starts over the 12-month study period than patients in the control group, with no differences in anti-hypertensive medication class changes. There were no significant reductions in SBP between baseline, 6 months, and 12 months in any group. This is in contrast to the 2010 Edelman et al trial that found improvements in blood pressure but not in Alc at the end of their group medical visits. ${ }^{12}$ However, whereas their participants at baseline had both poor glycemic and blood pressure 


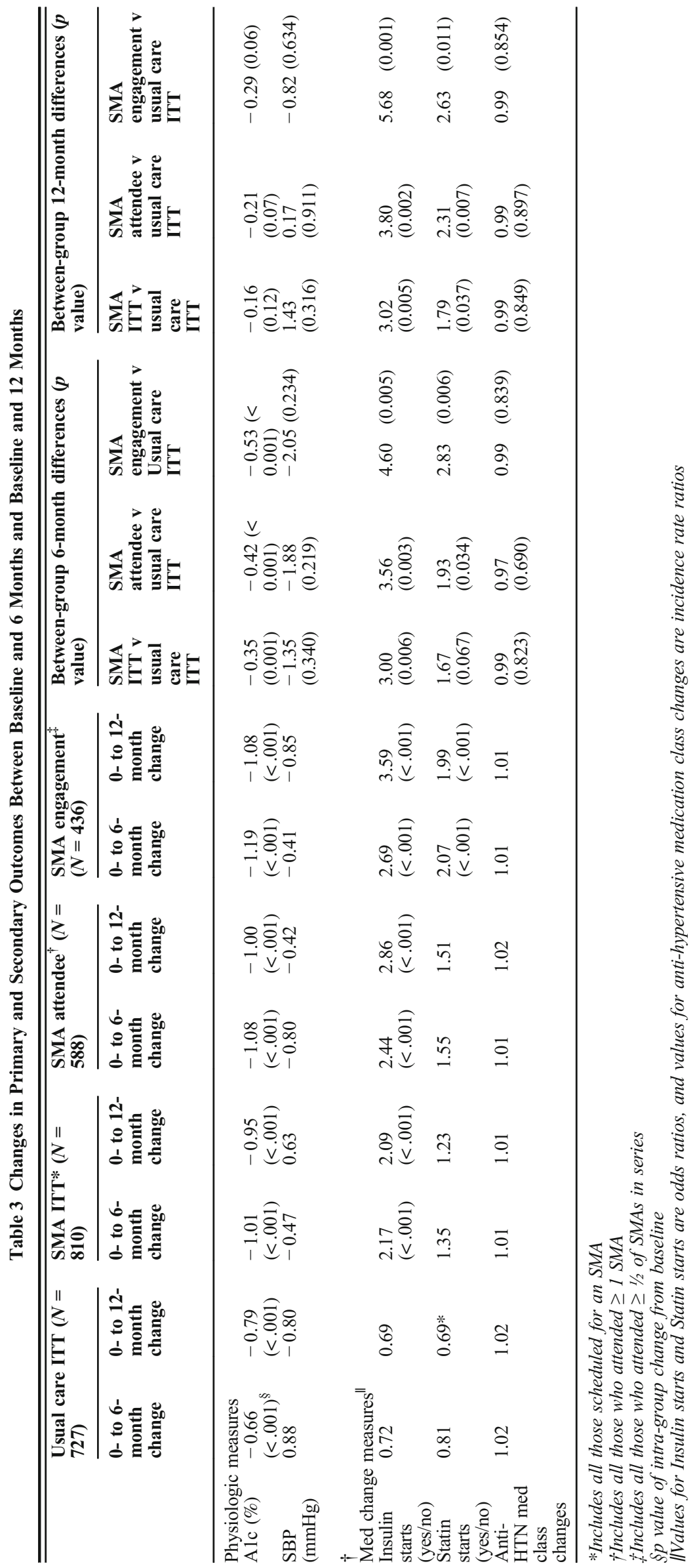


control, our participants' baseline blood pressure control was relatively good, with mean baseline SBPs of $<140 \mathrm{mmHg}$. Thus, they and the SMA facilitators could focus more on efforts to improve glycemic control. Moreover, the sessions in that trial were more didactic, whereas ours were designed to be patient-directed, interactive, incorporating action planning to set behavioral goals at each session, approaches that have been found to contribute to improved glycemic control. ${ }^{20,23-}$ 25,35

This evaluation of actual implementation of diabetes SMAs extends prior findings from efficacy trials that found significant reductions in A1c at the conclusion of SMAs compared with usual care. ${ }^{7-11,13-16}$ In many areas of clinical innovation, programs found to be efficacious in RCTs conducted under well-controlled conditions are not effective when implemented under real-life conditions. ${ }^{36-38}$ While relative A1c improvements at 6 months follow-up in our evaluation were more modest than improvements in some efficacy trials, they were still sufficiently greater to confer clinical benefit.

Our findings of improved glycemic control at completion of this model of diabetes SMAs are encouraging. Yet, as is the case for many short-term programs, there was no longer a significant difference between SMA and control patients 89 months after the end of the SMA series. Relative improvements in A1c at 12 months among participants who attended at least half of SMA sessions approached statistical significance ( $p$ $=.06$ ), but did not achieve it. SMA participants' greater uptake in statins and insulin was sustained at 12 months, and participation in SMAs may have other benefits, such as greater access to and more appropriate health care use, lower expenditures and hospitalizations over time as found in Jackson et al's follow up study, ${ }^{17}$ and improved patient-centered outcomes. We are exploring some of these outcomes in other research.

An important question is how to sustain the comparative gains achieved through SMAs. ${ }^{39}$ Our findings suggest that ongoing SMAs may be needed to sustain this intervention's relative advantages. Trento et al. found continued gains in A1c and other diabetes outcomes over the course of a 5-year group diabetes care model. ${ }^{8}$ We had hypothesized that offering participants at the first SMA the P2P program might be an effective and scalable approach to maintaining gains. Very few SMA participants elected to participate in this option, however. It is possible that uptake would have been greater if the option of P2P had been offered again later in the SMA series once participants had the chance to get to know each other and establish rapport and trust. Anecdotally, a number of SMA participants reported having contact outside of the SMA sessions, but we did not examine this systematically. In any case, the low uptake of P2P suggests that this approach as currently designed - while effective in efficacy trials ${ }^{18}$ - did not translate well in real-life implementation in these five health systems. Implementation in usual clinical practice of other promising models, including phone calls, emails, texts, or other modalities of proactive outreach by trained peer coaches or other health care team members, should be rigorously evaluated.

Our study has limitations. First, all study sites, though geographically diverse, were VA healthcare systems, and patients were predominantly white and men. Results may not generalize to other settings and populations. Second, as an implementation study, all clinical outcomes were gathered from EHRs. Accordingly, we had lower rates of follow-up clinical data and larger windows for capture of data than if we were bringing participants in to collect data by a research team. Our sensitivity analyses to address this deficiency, however, found no significant differences in findings.

In conclusion, our evaluation of implementation of diabetes SMAs within routine clinical programming across five healthcare systems demonstrated improved A1Cs soon after the SMA series' conclusion. SMAs did not improve A1c levels compared with usual care at 12 months, and few patients were interested in the supplementary peer support intervention. These findings add to the evidence that short-term SMAs are effective for diabetes, including when implemented as part of routine clinical care. There remains a great need to test longer term interventions and scalable follow-up programs to help sustain relative improvements achieved through short-term programs.

Supplementary Information The online version contains supplementary material available at https://doi.org/10.1007/s11606-02006570-y.

Acknowledgments: We express our sincere thanks to all clinicians involved in providing SMAs and P2P to our study participants. Michele Heisler takes full responsibility for the work as a whole.

Corresponding Author: Michele Heisler, MD, MPA; North Campus Research Complex, University of Michigan, Ann Arbor, MI, USA (e-mail: mheisler@umich.edu).

Authors' contributions All authors participated in the design of the study. MH conceived of the study, created the application that succeeded in obtaining the funding from VA Health Services Research \& Development (HSR\&D), and is the principal investigator of the study. JB managed study staff, organized meetings, provided staff training, managed study data, and completed all regulatory documents. DZ, JC, AG, SJ, LS, AT, and WWoversaw local implementation of the protocol and ensured study compliance. MH drafted the manuscript. All authors edited and approved the final manuscript.

Funding This research was financially supported a Grant from the Veteran Affairs Health Services Research and Development Service (IIR 15-321) and by Grant Number P30DK092926 (MCDTR) from the National Institute of Diabetes and Digestive and Kidney Diseases.

\section{Compliance with ethical standards:}

Conflict of interest: The authors declare that they do not have a conflict of interest.

Disclaimer: The views expressed in this paper are those of the authors and do not necessarily represent the views of the Department of Veterans Affairs.

Open Access This article is licensed under a Creative Commons Attribution 4.0 International License, which permits use, sharing, 
adaptation, distribution and reproduction in any medium or format, as long as you give appropriate credit to the original author(s) and the source, provide a link to the Creative Commons licence, and indicate if changes were made. The images or other third party material in this article are included in the article's Creative Commons licence, unless indicated otherwise in a credit line to the material. If material is not included in the article's Creative Commons licence and your intended use is not permitted by statutory regulation or exceeds the permitted use, you will need to obtain permission directly from the copyright holder. To view a copy of this licence, visit http://creativecommons. org/licenses/by/4.0/.

\section{REFERENCES}

1. Ligthart S, wan Herpt TTW, Leening MJG, et al. Lifetime risk of developing impaired glucose metabolism and eventual progression from prediabetes to type 2 diabetes: a prospective cohort study. Lancet Diabetes Endocrinol. 2016;4(1):44-51.

2. Davies MJ, D'Alessio DA, Fradkin J, et al. Management of Hyperglycemia in Type 2 Diabetes, 2018. A Consensus Report by the American Diabetes Association (ADA) and the European Association for the Study of Diabetes (EASD). Diabetes Care 2018;41(12):2669-2701.

3. Pillay J, Armstrong MJ, Butalia S, et al. Behavioral Programs for Type 2 Diabetes Mellitus: A Systematic Review and Network Meta-analysis. Ann Intern Med 2015; 163:848-860.

4. Bodenheimer T, Handley MA. Goal-setting for behavior change in primary care: an exploration and status report. Patient Educ Couns 2009;76(2):174-180.

5. Kirsh S, Watts S, Schaub K, et al. Training Manual, VA Shared Medical Appointments for Patients with Diabetes: Maximizing Patient \& Provider Expertise to Strengthen Care Management. 2008. http://www.queri.research.va.gov/tools/diabetes/shared-med-appt.pdf. Accessed 1 August 2016.

6. Kirk JK, Devoid HM, Strickland CG. Educational Strategies of Diabetes Group Medical Visits: A Review. Curr Diabetes Rev 2018;14(3):227-236.

7. Taveira TH, Friedmann PD, Cohen LB, Dooley AG, Khatana SA, Pirraglia PA, Wu WC. Pharmacist-led group medical appointment model in type 2 diabetes. Diabetes Educ 2010; 36(1): 109-17.

8. Trento, M, Passera P, Borgo E et al. 5-year randomized, controlled study of learning, problem solving ability, and quality of life modifications in people with type 2 diabetes managed by group care. Diabetes Care 2004; 27: 670-675.

9. Cohen LB, Taveira TH, Khatana SAM, Dooley AG, Pirraglia PA, Wu WC. Pharmacist-led shared medical appointments for multiple cardiovascular risk reduction in patients with type 2 diabetes. Diabetes Educ 2011;37(6):801-812.

10. Sadur CN, Moline N, Costa M, et al. Diabetes management in a health maintenance organization: efficacy of care management using cluster visits. Diabetes Care 1999;22:2011-2017.

11. Trento M, Passera $\mathbf{P}$, Tomalino $\mathbf{M}$, et al. Group visits improve metabolic control in type 2 diabetes: a 2-year follow-up. Diabetes Care 2001;24:995-1000.

12. Edelman D, Fredrickson SK, Melnyk SD, Coffman CJ. Medical clinics versus usual care for patients with both diabetes and hypertension: a randomized trial. Ann Intern Med 2010; 152: 689-696.

13. Edelman D, Gierisch JM, McDullfe JR, Oddone E, Williams, JW. Shared medical appointments for patients with diabetes mellitus: a systematic review. J Gen Intern Med 2015; 30: 99-106.

14. Housden LM, Wong ST. Using group medical visits with those who have diabetes: examining the evidence. Curr Diab Rep. 2016;16:134.https:// doi.org/10.1007/s11892-016-0817-4

15. Menon K, Mousa A, de Courten MP, Soldatos G, Egger G, de Courten B Shared medical appointments may be effective for improving clinical and behavioral outcomes in type 2 diabetes: a narrative review. Front Endocrinol (Lausanne). 2017;8:263. https://doi.org/10.3389/fendo.2017.00263

16. Vaughan EM, Johnston CA, Arlinghaus KR, Hyman DJ, Foreyt JP. A Narrative Review of Diabetes Group Visits in Low-Income and Underserved Settings. Curr Diabetes Rev 2019;15:372. https://doi.org/10. 2174/1573399814666181112145910

17. Jackson GJ, Edelman D, Olsen MK et al. Benefits of participation in diabetes group visits after trial completion. JAMA Int Med 2013; 173:590-592.

18. Heisler M, Vijan S, Makki F, Piette J. Diabetes Control with reciprocal peer support versus nurse care management: A randomized trial. Ann Intern Med 2010; 153:507-515.
19. Heisler, M., Burgess, J., Cass, J. et al. The Shared Health Appointments and Reciprocal Enhanced Support (SHARES) study: study protocol for a randomized trial. Trials 18, 239 (2017). https://doi.org/10.1186/ s13063-017-1959-7

20. Kirsh SR. Aron DC, Johnson KD et al. A realist review of shared medical appointments: How, for whom, and under what circumstances do they work? BMC Health Serv Res 2017; 17:113.

21. Drake, C Kirk JK, Buse JB, et al. Characteristics and Delivery of Diabetes Shared Medical Appointments in North Carolina. N C Med J 2019; 80:261-268.

22. Kirsh, Susan R., Renée H. Lawrence, and David C. Aron. Tailoring an intervention to the context and system redesign related to the intervention: A case study of implementing shared medical appointments for diabetes. Implement Sci 3.1 (2008): 34.

23. Miller WR, Rollnick S. Motivational Interviewing: Helping People Change. NY: Guilford; 2012.

24. Resnicow $\mathbf{K}$, McMaster $\mathbf{F}$. Motivational Interviewing: moving from why to how with autonomy support. Int $J$ Behav Nutr Phys Act 2012;9:19.

25. Williams GC, Freedman ZR, Deci EL. Supporting autonomy to motivate patients with diabetes for glucose control. Diabetes Care 1998;21:164451.

26. Gelman, Andrew, and Jennifer Hill. Data analysis using regression and multilevel/hierarchical models. Cambridge university press: Cambridge, 2006.

27. Hedeker, D. (2005). Generalized Linear Mixed Models. In Encyclopedia of Statistics in Behavioral Science (eds B.S. Everitt and D.C. Howell). https://doi.org/10.1002/0470013192.bsa251

28. Lovric M. (eds) International Encyclopedia of Statistical Science. Springer, Berlin, Heidelberg. https://doi.org/10.1007/978-3-642-04898-2

29. Vargha, A., \& Delaney, H. D. (2000). A Critique and Improvement of the CL Common Language Effect Size Statistics of McGraw and Wong. J Educ Behav Stat, 25(2), 101-132. https://doi.org/10.3102/ 10769986025002101

30. Abadie, A. and Imbens, G.W. (2016), Matching on the Estimated Propensity Score. Econometrica, 84: 781-807. https://doi.org/10. 3982/ECTA11293

31. Sterne Jonathan A C, White Ian R, Carlin John B, Spratt Michael, Royston Patrick, Kenward Michael G et al. Multiple imputation for missing data in epidemiological and clinical research: potential and pitfalls BMJ 2009; 338 :b2393. https://doi.org/10.1136/bmj.b2393

32. Carpenter JR, Kenward MG. Multiple Imputation and its Application. Hoboken, NJ: John Wiley \& Sons; 2013.

33. Haynes W. (2013) Benjamini-Hochberg Method. In: Dubitzky W., Wolkenhauer O., Cho KH., Yokota H. (eds) Encyclopedia of Systems Biology. Springer, New York, NY. https://doi.org/10.1007/978-1-44199863-7

34. UKPDS, Intensive blood-glucose control with sulphonylureas or insulin compared with conventional treatment and risk of complications in patients with type 2 diabetes (UKPDS 33). UK Prospective Diabetes Study (UKPDS) Group. Lancet, 1998. 352(9131):837-8.

35. Kwan, Bethany M, Dickinson LM, Glasgow RE, et al. "The Invested in Diabetes Study Protocol: a cluster randomized pragmatic trial comparing standardized and patient-driven diabetes shared medical appointments. Trials. 2020; 21:65.

36. Wu WC, Taveira TH, Jeffery S, Jiang L, Tokuda L, Musial J, Cohen LB, Uhrle F. Costs and effectiveness of pharmacist-led group medical visits for type-2 diabetes: A multi-center randomized controlled trial. PLoS One. 2018;13(4):e0195898. https://doi.org/10.1371/journal.pone.0195898. eCollection 2018.

37. Heisler M, Hofer TP, Schmittdiel JA, Selby JV, Klamerus ML, Bosworth HB, Bermann M, Kerr EA. Improving blood pressure control through a clinical pharmacist outreach program in diabetes patients in two-high performing health systems: The Adherence and Intensification of Medications (AIM) cluster randomized controlled effectiveness trial. Circulation. 2012; 125(23):2863-2872.

38. Damschroder LJ, Lowery JC. Evaluation of a large-scale weight management program using the consolidated framework for implementation research (CFIR). Implement Sci 2013;8:51.

39. Taveira $\mathbf{T H}, \mathbf{W u} \mathbf{W C}$. Interventions to maintain cardiac risk control after discharge from a cardiovascular risk reduction clinic: a randomized controlled trial. Diabetes Res Clin Pract 2014;105(3):327-335. https:// doi.org/10.1016/j.diabres.2014.05.013

Publisher's Note: Springer Nature remains neutral with regard to jurisdictional claims in published maps and institutional affiliations. 